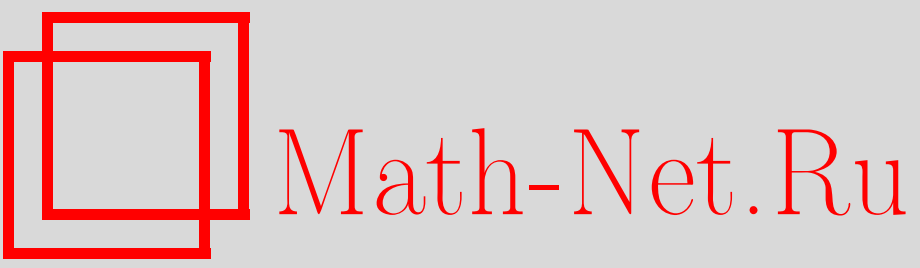

В. В. Чеканов, Н. В. Кандаурова, В. С. Чеканов, М. Ю. Шевченко, С. Р. Мирзаханов, Математическое моделирование автоволновых процессов в ячейке с наноструктурированной жидкостью, Итоги науки и техн. Сер. Соврем. мат. и ее прил. Темат. обз., 2019, том 165, 80-87

DOI: https://doi.org/10.36535/0233-6723-2019-165-80-87

Использование Общероссийского математического портала Math-Net.Ru подразумевает, что вы прочитали и согласны с пользовательским соглашением

http://www.mathnet.ru/rus/agreement

Параметры загрузки:

IP : 54.198 .187 .58

26 апреля 2023 г., 14:19:08 


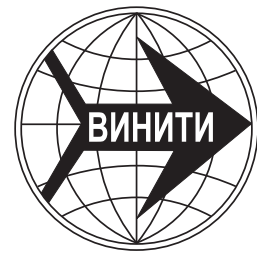

ИТОГИ НАУКИ И ТЕХНИКИ.

Современная математика и ее приложения.

Тематические обзоры.

Том 165 (2019). С. 80-87

DOI: $10.36535 / 0233-6723-2019-165-80-87$

УДК 517.958; 538.97

\title{
МАТЕМАТИЧЕСКОЕ МОДЕЛИРОВАНИЕ АВТОВОЛНОВЫХ ПРОЦЕССОВ В ЯЧЕЙКЕ С НАНОСТРУКТУРИРОВАННОЙ ЖИДКОСТЬЮ
}

\author{
(c) 2019 г. В. В. ЧЕКАНОВ, Н. В. КАНДАУРОВА, В. С. ЧЕКАНОВ, \\ М. Ю. ШЕВЧЕНКО, С. Р. МИРЗАХАНОВ
}

\begin{abstract}
АннотАция. Найдено решение математической модели автоволнового процесса в тонком приэлектродном слое магнитной жидкости. Модификация базовой системы ФитцХью-Нагумо, взятой за основу математической модели, позволила получить результаты, аналогичные натурному эксперименту. В качестве критерия адекватности модели выступает визуальное соответствие эксперименту, а также наличие в решении основных явлений, наблюдаемых в автоволновом процессе, таких как ревербераторы, пейсмекеры, огибание препятствий и др.
\end{abstract}

Ключевые слова: автоволна, самоорганизация, магнитная жидкость, модель ФитцХьюНагумо, математическое моделирование.

\section{MATHEMATICAL MODELING OF AUTOWAVE PROCESSES IN A CELL WITH NANOSTRUCTURED LIQUID}

(c) 2019 V. V. CHEKANOV, N. V. KANDAUROVA, V. S. CHEKANOV, M. YU. SHEVCHENKO, S. R. MIRZAHANOV

\begin{abstract}
In this paper, a solution of the mathematical model of an autowave process in a thin nearelectrode layer of a magnetic fluid is found. A modification of the FitzHugh-Nagumo system taken as the basis of the mathematical model made it possible to obtain results similar to a full-scale experiment. As a criterion for the adequacy of the model, we use a visual correspondence to the experiment and the presence in the solution of typical phenomena observed in the autowave process, such as reverbs, pacemakers, encircling of obstacles, etc.
\end{abstract}

Keywords and phrases: autowave, self-organization, magnetic fluid, FitzHugh-Nagumo model, mathematical modeling.

AMS Subject Classification: 90-08

1. Введение. Активные среды в течение нескольких последних десятилетий вызывают интерес как у исследователей-экспериментаторов, так и специалистов в области математического моделирования. Интерес этот вызван прежде всего тем, что многие процессы в химических, биологических системах, нейронных сетях и человеческом мозге подчиняются одинаковым законам. По определению авторов [5] «основные формы кооперативного поведения, свойственные живым организмам, имеют свои аналоги среди неорганических систем».

Работа выполнена в рамках госзадания (базовая часть) № 3.5385.2017/8.9 «Экспериментальное исследование и математическое моделирование межфазных и приповерхностных явлений в тонкой пленке наноструктурированной магнитной жидкости». 
Настоящая статья посвящена математическому моделированию процесса самоорганизации в коллоидной системе - тонком слое концентрированной магнитной жидкости. В [6] было показано, что тонкий приэлектродный слой является активной средой, в которой наблюдались автоволны (см. [7]). Подробное описание экспериментальной установки и техники проведения эксперимента по наблюдению автоволн в магнитной жидкости можно найти в $[6,7]$.

Структуры, возникающие в тонком приэлектродном слое концентрированной магнитной жидкости в результате автоволнового процесса, очень удобны для экспериментального изучения. Процесс идет в обычных лабораторных условиях, не иссякает и может протекать неограниченно долго. Движение волн сопровождается изменением цвета поверхности. Время протекания процесса достаточно быстрое (импульс возбуждения движется со скоростью несколько миллиметров в секунду), волны и образовавшиеся структуры можно наблюдать невооруженным глазом (размер поля наблюдения $-6 \times 8 \mathrm{~cm})$.

Первая математическая модель автоволнового процесса в магнитной жидкости предложена в [8]. Эта модель имела существенные недостатки, так как состояла из одного дифференциального уравнения в частных производных параболического типа, а сама среда рассматривалась как бистабильная. Далее, в [9] было показано, что расматриваемая среда является возбудимой, поэтому для ее описания можно применить базовую модель ФитцХью-Нагумо. Новая модель содержала два уравнения, первое из которых описывало быстрый процесс (активацию), а второе медленный (ингибирование). Однако решение модели, согласующееся с натурными экспериментами получено не было.

Цель настоящей работы - показать адекватность математической модели [5], получив решение в среде COMSOL Multiphysics, которое согласуется с экспериментальными данными.

2. Описание физической модели. Магнитная жидкость представляет собой сложную коллоидную систему, состоящую из ферримагнитных частиц нанометровых размеров, находящихся во взвешенном состоянии в несущей жидкости, в качестве которой обычно выступает органический растворитель (керосин) или вода. Для обеспечения устойчивости такой жидкости ферримагнитные частицы связываются с поверхностно-активным веществом (ПАВ), образующим защитную оболочку вокруг частиц и препятствующим их слипанию. В нашей работе была использована магнитная жидкость типа «магнетит в керосине». Средний размер частиц магнетита - 10 нм, в качестве стабилизатора (ПАВ) использовалась олеиновая кислота.

Физический механизм автоволнового процесса в магнитной жидкости состоит в следующем: в электрическом поле заряженные частицы магнетита вследствие электро- и диполофореза движутся к ближайшему электроду и образуют около него плотно упакованный слой толщиной порядка 150 нм. Между слоем частиц и электродом образуется тонкая прослойка - так называемый структурномеханический барьер, который состоит из диэлектрика - смеси керосина и молекул олеиновой кислоты (поверхностно-активного вещества, окружающего частицы магнетита). Толщина этого барьера порядка 5 нм, но он препятствует соприкосновению частиц магнетита и электрода и разрядке частиц. С ростом приэлектродного слоя частиц в структурномеханическом барьере растет напряженность, и при ее критическом значении $E \sim 10^{7}$ В/м диэлектрик становится проводящим в результате смещения константы химического равновесия, при котором резко увеличивается число ионов. Частицы магнетита перезаряжаются и начинают движение от электрода - проходит одиночная плоская автоволна.

Считая среду возбудимой, предположим, что она состоит из множества элементарных площадок приэлектродного слоя. По определению возбудимой среды каждый такой участок является автономным источником энергии. Энергия накапливается за счет так называемой поляризационной емкости приэлектродного слоя частиц (см. [10]). Элементы возбудимой среды взаимодействуют между собой, передавая энергию электрического и гидродинамического взаимодействия от одного к другому. Так передается импульс возбуждения. Этот процесс аналогичен взаимодействию нелинейных связанных осцилляторов с короткой связью, где каждый осциллятор связан только с несколькими соседними. Таким образом, физический смысл разрабатываемой модели автоволнового процесса заключается в рассмотрении системы связанных нелинейных осцилляторов. 
Элемент возбудимой среды может находиться в трех состояниях: покой, возбуждение и рефрактерность.

Локальные элементы, из которых состоит исследуемая активная среда (элементы приэлектродного слоя) имеет «единственное выделенное состояние покоя, устойчивое по отношению к достаточно слабым внешним воздействиям» (см. [5]). Это состояние среды, когда приэлектродный слой уже образовался (напряжение на электродах меньше критического), но автоволновой процесс еще не начался.

Когда напряжение на электродах становится больше критического, элемент возбуждается и становится активным, через элементарный конденсатор происходит разряд. Это состояние возбуждения, или так называемый активный переход, после которого элементарный участок слоя перестает существовать, потому что слой на этом локальном участке разрушается. При этом по поверхности проходит одиночный волновой фронт.

Рефрактерность - это состояние, когда среда накапливает энергию и не способна к проведению нового импульса, наступает оно после возбуждения. Среда должна восстановить свои свойства за счет поступающей извне энергии и подготовиться к проведению следующего импульса. Энергия от внешнего источника продолжает поступать, частицы магнетита движутся к ближайшему электроду, образуя систему из последовательно соединенных конденсаторов: первый электрод структурномеханический барьер, слой плотноупакованных частиц-магнитная жидкость низкой концентрации - слой плотноупакованных частиц - структурномеханический барьер - второй электрод. Период рефрактерности соответствует зарядке конденсаторов, одной пластиной которых является электрод, а другую составляет приэлектродные слои.

3. Описание математической модели. Для моделирования спиральных волн, пейсмекеров, огибания препятствия воспользуемся базовой моделью ФитцХью-Нагумо, которая описывает автоволновой процесс в возбудимых средах. Она позволяет широко настраивать параметры автоволнового процесса и изменять характеристики его протекания.

Модель состоит из двух уравнений, первое уравнение из которых описывает «быстрый» процесс» - перезарядка частиц в приэлектродном слое после возрастания проводимости структурномеханического барьера. Второе уравнение описывает медленный процесс - изменение заряда в приэлектродном слое, когда в нем накапливаются заряженные частицы магнетита:

$$
\begin{aligned}
& \frac{\partial V}{\partial t}+\frac{\partial^{2} V}{\partial t^{2}}=D \Delta V-V^{3}+V-I, \\
& \frac{\partial I}{\partial t}=\varepsilon(V+\alpha-b I)
\end{aligned}
$$

здесь $V$ - потенциал действия, т.е. функция, зависящая от напряженности поля в тонком приэлектродном слое магнитной жидкости (активатор); $I$ - восстанавливающая переменная, т.е. ингибитор: величина, которая нарастает с нарастанием $V$ и убывает по мере собственного накопления, эта функция связана с изменением заряда в приэлектродном слое; $D$ - коэффициент диффузии активатора; $\varepsilon$ - малый параметр, представляющий собой отношение времени прохождения одиночного импульса и времени образования приэлектродного слоя; $\alpha$ и $b$-параметры. В зависимости от значений параметров элемент среды может находиться либо в автоколебательном, либо в возбудимом режиме. В случае возбудимой среды колебания возникают благодаря обратной связи, которая обеспечивается периодическими граничными условиями (см. [3]).

\section{4. Результаты моделирования в среде COMSOL Multiphysics. Визуализация реше-} ний. Выбор в качестве среды моделирования COMSOL Multiphysics 5.2 (CM) в основном связан с тем, что используемый в СМ метод конечных элементов позволяет учитывать непостоянство параметров внутри элементов рассматриваемой системы и существующую нелинейность.

В качестве критерия адекватности предложенной модели выступало визуальное соответствие натурному эксперименту, а также наличие основных явлений, наблюдаемых в автоволновом процессе, таких как ревербераторы, пейсмекеры, огибание препятствий и т. д. 
4.1. Моделирование ревербераторов. Если в силу каких-то условий происходит разрыв плоской волны возбуждения, то образуется спиральная волна (ревербератор, автоволновой вихрь) (см. [1]).

В спиральной волне обрыв ее фронта вращается вокруг фиксированной точки - центра спирали - по окружности определенного радиуса. Внутрь круга, ограниченного этой окружностью и называемого ядром, автоволна не проникает. В возбудимой среде ядро остается невозбужденным. Форма спиральной волны вдали от центра вращения близка к эвольвенте окружности (см. [2]). Экспериментальные результаты исследования спиральных волн в приэлектродном слое магнитной жидкости описаны в [9].

Модель была реализована в виде компьютерной симуляции. Получено решение для одно, двухи трехрукавных ревербераторов (рис. 2, 3).

Создание модели ревербератора состоит из двух частей: задание плоской волны и ее обрыв. В случае единичного ревербератора горизонтальная плоская волна задается с помощью уравнения

$$
I=I_{0} \operatorname{th}\left(80\left(y-y_{0}\right)\right),
$$

где $I_{0}$ - начальное значения функции $I(0,3), x_{0}$ и $y_{0}$ - координаты центра ревербератора. Для преобразования плоской волны в однорукавный ревербератор (рис. 1) необходим обрыв волны. Он задается с помощью прямоугольной области с высоким значением активатора:

$$
V=V_{0}, \quad x>x_{0}, y>y_{0},
$$

где $V_{0}$ - начальное значение функции $V(I), x_{0}$ и $y_{0}$-координаты центра ревербератора. В этой области не происходит формирования волны синхронно с остальным фронтом, а образуется обрыв. Плоская волна с обрывом начинает изгибаться вокруг него. Таким образом, формируется однорукавный спиральный ревербератор в точке $\left(x_{0}, y_{0}\right)$ (см. рис. 1$)$.

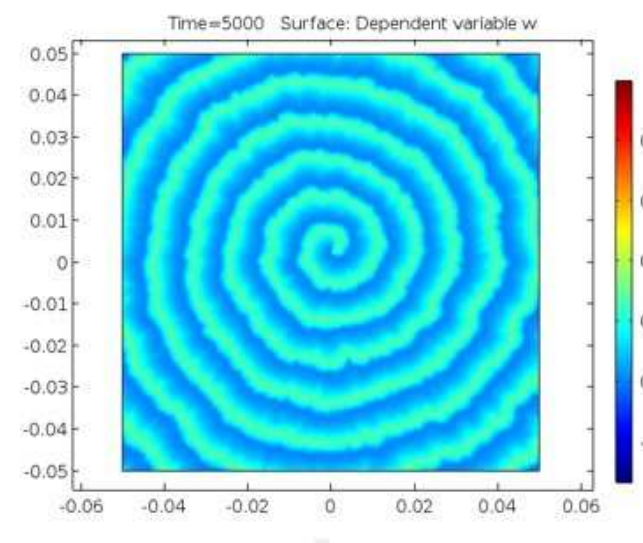

a

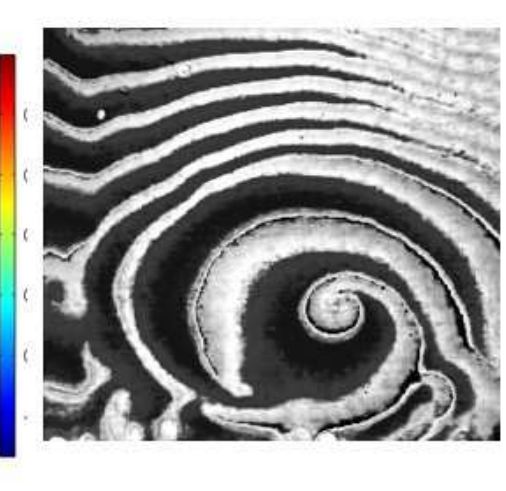

6

Рис. 1. Слева - результат моделирования развития однорукавного ревербератора; справа - результат натурного эксперимента. Цветом показано значение величины активатора.

Множественные обрывы формируют множественные ревербераторы попеременной направленности. Если задать два обрыва волны:

$$
V=V_{0}, \quad x^{2}>\left(x_{0}+\frac{d}{2}\right)^{2}, \quad y>y_{0},
$$

где $d$-расстояние между центрами ревербераторов, то получится два разнонаправленных ревербератора.

Для создания сонаправленных групп ревербераторов используется радиальное задание условий:

$$
V=V_{0} \sin (\operatorname{arctg} 2(y, x) N), q q q I=I_{0}\left(\cos \left(\operatorname{arctg} 2(y, x) \frac{N}{2}-0.25\right)\right)^{4}
$$


где $N$ - количество ревербераторов. Для случаев $N=2$ и $N=3$ результаты приведены на рис. 2.

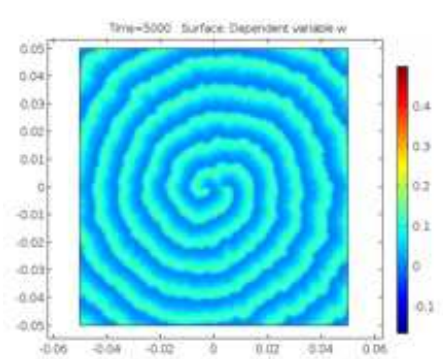

a

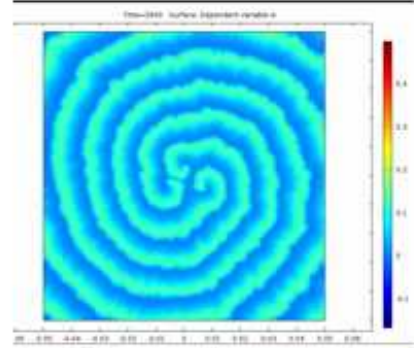

б

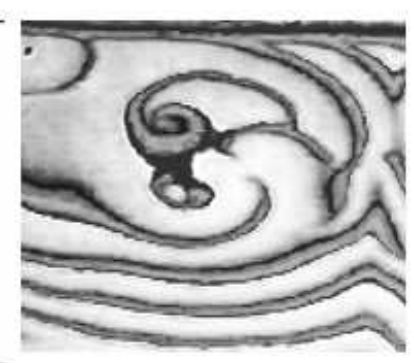

B

Рис. 2. Результат моделирования: слева - двухрукавный сонаправленный ревербератор, в центре - трехрукавный разнонаправленный ревербератор, справа - натурный эксперимент.

Из-за радиальных координат ширина волны слишком мала в центре и увеличивается по мере удаления от центра. Слишком широкие участки волны коллапсируют до размера, соответствующего свойствам среды, в то время как слишком узкие не распространяются, образуя обрыв волнового фронта.

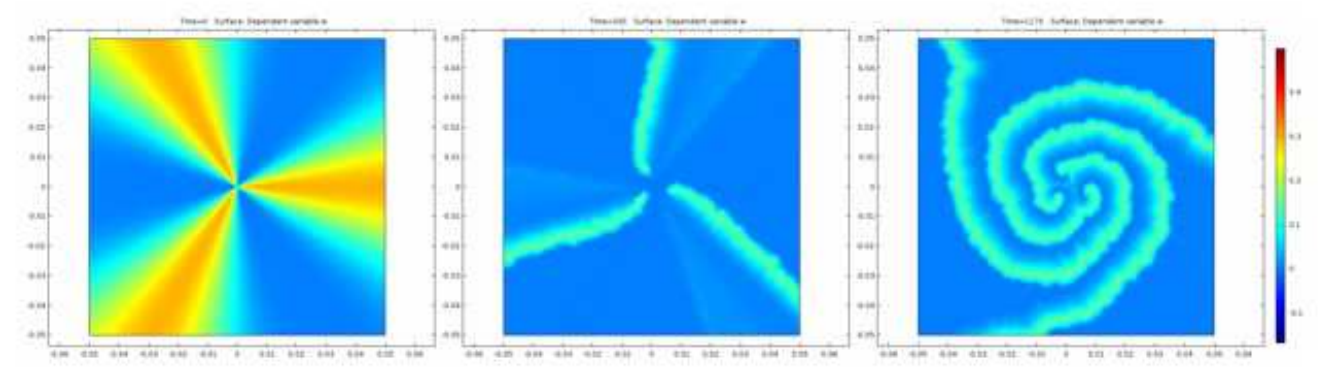

Рис. 3. Моделирование трехрукавного ревербератора (пошаговое).

В компьютерном эксперименте мы столкнулись с проблемой неуправляемости обрыва фронта, который позволяет задавать количество и направление ревербераторов в симулируемой системе. Дополнительные симуляции позволили выяснить, что произвольные обрывы фронта происходят из-за слишком большого различия между толщиной волны и размером сетки. Поскольку размер симуляции напрямую зависит от размера расчетной сетки, это соотношение остается одинаковым при разных размерах ячейки. Изменение коэффициентов уравнений в системе 1 позволило получить более широкий и стабильный фронт волны. Изменяя количество обрывов волны и их направление, можно моделировать явления, связанные с граничными условиями.

В полученных моделях была обнаружена анизотропия распространения волн, которая проявлялось в появлении углов на распространяющихся волнах (рис. 5). Причиной анизотропии служила сетка разбиения, поскольку стандартное разбиение квадратной ячейки приводит к неравной концентрации точек в разных направлениях. Для исправления этого дефекта метод генерации сетки разбиения был заменен на метод Делоне, поскольку он позволяет получить сетку с более высокой изотропностью.

4.2. Моделирование пейсмекеров. Источником автоволн в активной среде может служить область, называемая пейсмейкером, испускающая автоволны с некоторым периодом. Пейсмейкер создает в среде картину в виде кольцевых волн, расходящихся из центра. Период пейсмейкера 

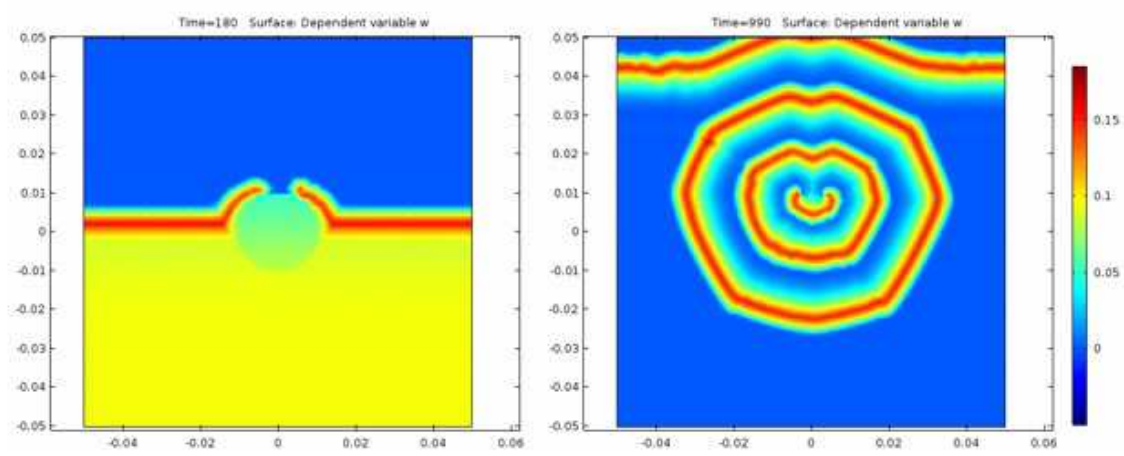

Рис. 4. Двойной обрыв волны и анизотропия распространения фронта волны.

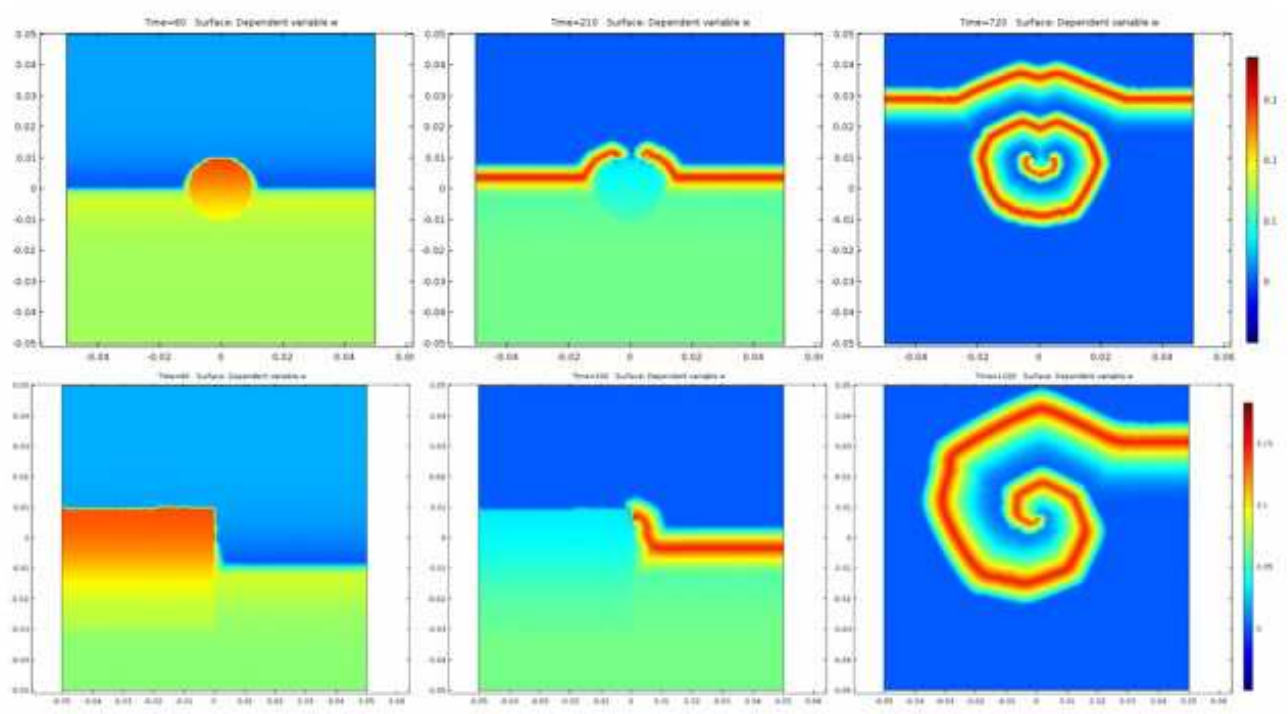

Рис. 5. Двойной обрыв волны и анизотропия распространения фронта волны.

определяется его собственными свойствами и может быть произвольным. При наличии в среде нескольких пейсмейкеров с разными периодами низкочастотные подавляются высокочастотными (см. [4]).

В рамках используемой модели пейсмекер можно создать с помощью точечного источника с небольшим сдвигом. Частота пейсмекера, заданного точечным источником, зависит от размера сдвига этого источника.

На рис. 6а показаны результаты моделирования пейсмекера; видно, что компьютерная симуляция соответствуют экспериментальным результатам (рис. 6б).

4.3. Моделирование огибания препятствия. Для моделирования огибания препятствий система 1 была модифицирована. Данное явление сложно объяснить чисто геометрическими причинами: путь волны вдоль препятствия короче, чем на расстоянии от него. При равных скоростях распространения внешняя часть волны не должна обгонять внутреннюю. Факт огибания можно объяснить тем, что среда неоднородна вблизи препятствия и, следовательно, скорость волны 


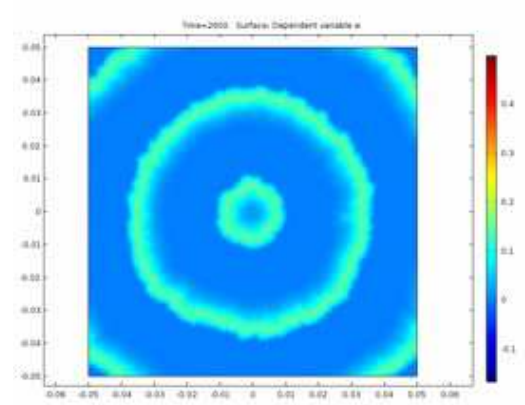

a

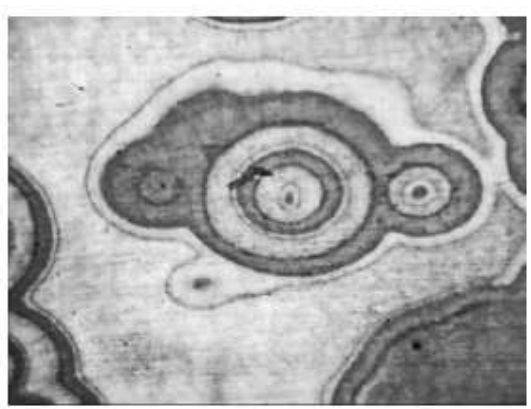

6

Рис. 6. Слева-результат моделирования единичного пейсмекера, справа - натурный эксперимент.

должна изменяться. Для описания этой неоднородности в уравнение системы был введен коэффициент замедления $\phi$. Модель огибания автоволной препятствия имеет вид:

$$
\begin{aligned}
& \frac{\partial V}{\partial t}+\frac{\partial^{2} V}{\partial t^{2}}=\frac{(\alpha-V)(V-1) V-I}{1+2 \phi}, \\
& \frac{\partial I}{\partial t}=\varepsilon\left(1+\frac{\phi}{1.5}\right)(\beta V-\gamma I-\delta) .
\end{aligned}
$$

Коэффициент ф зависит только от расстояния до ближайшей точки препятствия, и вычисляется по следующей формуле:

$$
\phi=\left(\frac{d}{r}\right)^{4} H, \quad d<r,
$$

где $d$ - расстояние до препятствия, $r$ - радиус действия функции замедления, $H$ - функция Хевисайда. Использование функции замедления позволяет моделировать огибание препятствия (рис. 7).

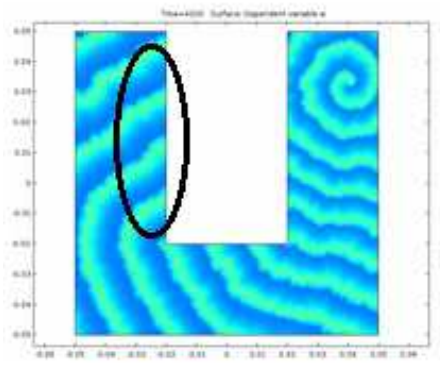

a

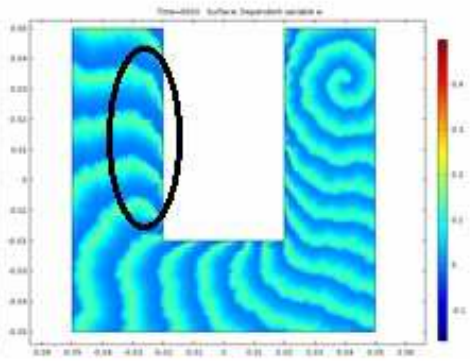

б

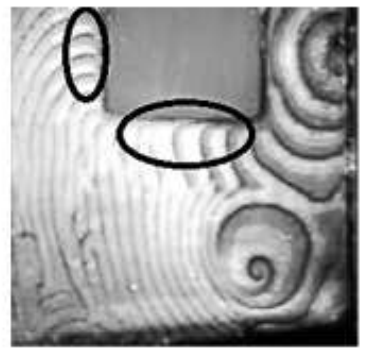

B

Рис. 7. Слева - симуляция огибания препятствия без функции $\phi$ в математической модели; в центре- симуляция огибания с функцией $\phi$ в модели, справа - натурный эксперимент.

Поскольку результаты симуляции огибания с функцией $\phi$ соответствуют натурному эксперименту (см. рис. 7), можно предполагать наличие неоднородности в свойствах среды вблизи препятствия.

5. Заключение. В работе проведено моделирование автоволнового процесса в магнитной жидкости средствами среды COMSOL Multiphysics 5.2. Рассмотрена математическая модель и ее модификации для различных автоволновых режимов. Получены решения для спиральных волн, 
пейсмекеров, огибания препятствия, которые позволяют исследовать основные свойства автоволн.

\section{СПИСОК ЛИТЕРАТУРЫ}

1. Балаховский И. С. Некоторые режимы движения возбуждения в идеальной возбудимой ткани// Биофизика. - 1965. - 6. - С. 1063-1067.

2. Винер Н., Розенблют А. Проведение импульсов в сердечной мышце. Математическая формулировка проблемы проведения импульсов в сети связанных возбудимых элементов, в частности, в сердечной мышце// Кибер. сб. - 1961. - 3. - С. 3-56.

3. Слепнев А. В., Вадивасова Т. Е. Два вида автоколебаний в активной среде с периодическими граничными условиями// Нелинейная динамика. -2012 . -8 , № 3. - С. 497- 505 .

4. Елъкин Ю. Е. Автоволновые процессы// Мат. биол. биоинформ. $-2006 .-1$, № 1.

5. Лоскутов А. Ю., Михайлов А. С. Основы теории сложных систем. - М.-Ижевск: Регулярная и хаотическая динамика, 2007.

6. Chekanov $V . V$. Self-organization in the surface layer of the magnetic liquid. Mechanisms of autowave appearance// in: Proc. 35 Joint Meeting of the European Molecular Liquids Group and the Japanese Molecular Liquids Group. — Vienna: Springer, 2017. — P. 115-116.

7. Chekanov V. V., Kandaurova $N$. V.,Chekanov $V$. S. Phase autowaves in the near-electrode layer in the electrochemical cell with a magnetic fluid// J. Magnetism Magnet. Materials. — 2017. — 431. - P. 38-41.

8. Chekanov V. V., Iljuch P. M., Kandaurova N. V., Bondarenko E. A. Auto-waves in near-surface layer of magnetic fluid// J. Magnetism Magnet. Materials. — 2005. — 289. — P. 107-109.

9. Chekanov V. V., Kandaurova N. V., Chekanov V. S. Experimental study of the properties of awutowave sources (reverberators) in the magnetic fluid near-elecrode layer using reflected light interference// J. Magnetohydrodynamics. - 2017. - 53, № 3. - P. 495-500.

10. Kandaurova $N$. V, Chekanov V. V, Chekanov V. S. Effect of a near-surface nanolayer formation on the magnetic fluid electrical properties// J. Acta Techn. — 2018. - 63, № 1B. - P. 1-8.

Чеканов Владимир Васильевич

Северо-Кавказский федеральный университет, Ставрополь, Россия

E-mail: chknv1@yandex.ru

Кандаурова Наталья Владимировна

МИРЭА-Российский технологический университет, Ставрополь, Россия

E-mail: candaur18@yandex.ru

Чеканов Владимир Сергеевич

МИРЭА-Российский технологический университет, Ставрополь, Россия

Шевченко Михаил Юрьевич

ООО «Оптосистемы», Москва, Россия

E-mail: xpohoc@gmail.com

Мирзаханов Сергей Робертович

Северо-Кавказский федеральный университет, Ставрополь, Россия 